\title{
Low-noise femtosecond Cherenkov fiber laser, continuously tunable across the entire red- green-blue spectral range
}

\author{
Xiaomin Liu ${ }^{1, *}$, Jesper Lægsgaard ${ }^{2}$, Roman Iegorov ${ }^{3}$, Ask Svane $^{2}$, F. Ömer Ilday ${ }^{4,5}$, \\ Haohua $\mathrm{Tu}^{6}$, Stephen A. Boppart ${ }^{6}$, and Dmitry Turchinovich ${ }^{1,7, \dagger}$ \\ ${ }^{1}$ Max Planck Institute for Polymer Research, Ackermannweg 10, 55128 Mainz, Germany \\ ${ }^{2}$ DTU Fotonik, Technical University of Denmark, 2800 Kgs. Lyngby, Denmark \\ ${ }^{3}$ National Research Tomsk Polytechnic University, Institute of Power Engineering, 30 Lenin Avenue, \\ 634050 Tomsk, Russia \\ ${ }^{4}$ Department of Physics, Bilkent University, 06800 Ankara, Turkey \\ ${ }^{5}$ Department of Electrical and Electronics Engineering, Bilkent University, 06800 Ankara, Turkey \\ ${ }^{6}$ Biophotonics Imaging Laboratory, University of Illinois at Urbana-Champaign, Urbana, Illinois \\ 61801, USA \\ ${ }^{7}$ Fakultät für Physik, Universität DuiburgßEssen, Lotharstraße 1, 47048 Duisburg, Germany
}

\begin{abstract}
Low-noise Cherenkov wave conversion in a tapered photonic crystal fiber turns a standard, fixed-wavelength femtosecond laser into an extremely widely-tunable source. Here we show the continuous laser tunability across the entire redgreen-blue range.
\end{abstract}

The laser emission wavelength is normally pre-determined by the specific gain medium used. Arbitrary wavelength generation, which is crucial for numerous applications in technology, as well as in natural and life sciences, is one of the fundamental challenges in laser science. Present widely-used solutions include optical parametric generation employing highly elaborate solid state technology [1]; and spectrally-sliced supercontinuum (SC) [2], taking advantage of simpler fiber technology, but with a high inherent noise figure [3]. Here we demonstrate a simple and inexpensive fiber technology based on fiber-optic Cherenkov radiation (FOCR) in a tapered photonic crystal fiber (PCF), which allows one to resonantly convert the emission wavelength of a standard pulsed laser to a wide and continuous range of desired wavelengths, with very low inherent noise, and without mechanical adjustment of any of the elements of the laser. As a first demonstration of this technology, we present a femtosecond fiber laser continuously tunable across the entire red-green-blue spectral range [4].

The laser wavelength tunable scheme relies on the following facts. First of all, FOCR, which is also known as dispersive wave generation or non-solitonic radiation [5], in contrast to SC, can efficiently convert the wavelength of any standard pump pulsed laser to

\footnotetext{
* Corresponding author: liuxiaomin@mpip-mainz.mpg.de

${ }^{\dagger}$ Corresponding author: dmitry.turchinovich@uni-due.de
} 
a desired wavelength in a spectrally-isolated fashion, at modest pump pulse energies, and with low noise [6]. Secondly, as illustrated in Fig. 1(a), by controlling the PCF's lateral dimension, and thus the fiber dispersion profile as well as the FOCR phase-matching condition, the resulting FOCR wavelength can be easily tuned over a broad spectral range. Thirdly, as showed in Fig. 1(b), the FOCR generation only happens at the spatial point of maximal pump pulse compression in a fiber. Therefore, by combining all those three conditions, in a single tapered PCF whose dispersion profile varies along the fiber length, the generated FOCR wavelength is then determined by the local fiber dispersion properties at this point of maximum pump pulse compression. In such a way, the FOCR generation in one single tapered fiber establishes a direct link between the FOCR wavelength and the pump pulse properties - power, duration and chirp - which can be easily controlled.
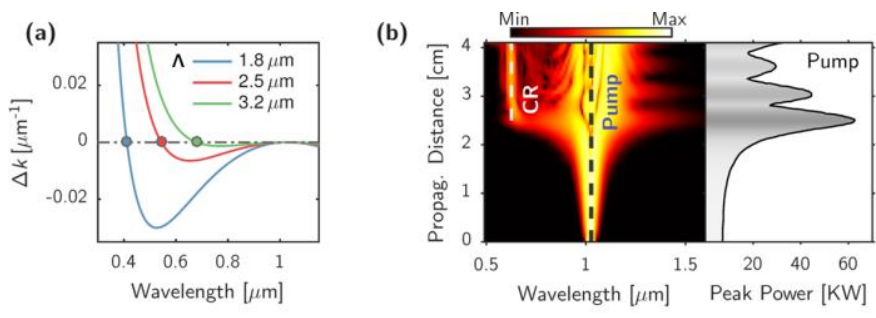

(c)

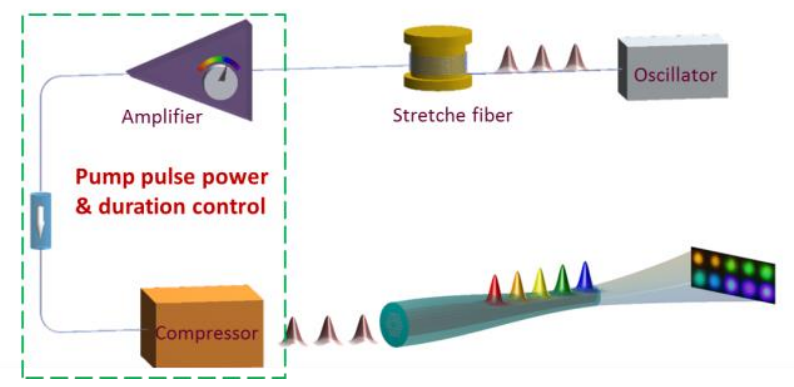

Fig. 1. Principle and experimental setup of tunable femtosecond fiber laser. (a) Phasematching curves of FOCR generation with different fiber dimensions. $\Lambda$ is the PCF pitch. (b) With a transform-limited Gaussian input pump pulse at $1035 \mathrm{~nm}$, the simulated spectrum evolution (left) and peak power evolution (right) of the pulse along a tapered PCF for FOCR generation at $580 \mathrm{~nm}$. (c) General layout of the tunable FOCR laser.

While many variations on this general principle can be envisioned, here we focus on the generation of short pulses tunable over the visible spectrum in the 400-600 nm range. Such a laser system as shown in Fig. 1(c) consists of two parts: a fixed-wavelength pump laser, and a tapered PCF. In this work, a standard mode-locked Yb-fiber laser with a center wavelength at $1035 \mathrm{~nm}$, pulse durations tunable from $90 \mathrm{fs}$ and maximum pulse energies in excess of $10 \mathrm{~nJ}$ is used as the pump laser. The pump pulse can be easily controlled by tuning the fiber amplifier and/or the compressor (green box in Fig. 1 (c)). The detail of the prepared tapered PCF can be found in Ref. [4].

As shown in Fig. 2(a), the broad laser wavelength tunability between 414 and $612 \mathrm{~nm}$ is achieved by electrical control of the fiber amplifier and the pulse compressor, and the experimental results closely match the theoretical predictions. Remarkably, even by adjusting only the pump power, the laser wavelength tunability over the $420-560 \mathrm{~nm}$ range, already covering much of the visible spectrum, can be obtained. Fig. 2(b) shows measured autocorrelation curves of the generated FOCR pulses which are in the range of about 100 fs. As one can see, the FOCR pulses are much shorter than what is typically produced by 
currently most-used spectrally sliced SC technology [7], which can benefit the applications such as nonlinear spectroscopy and microscopy.
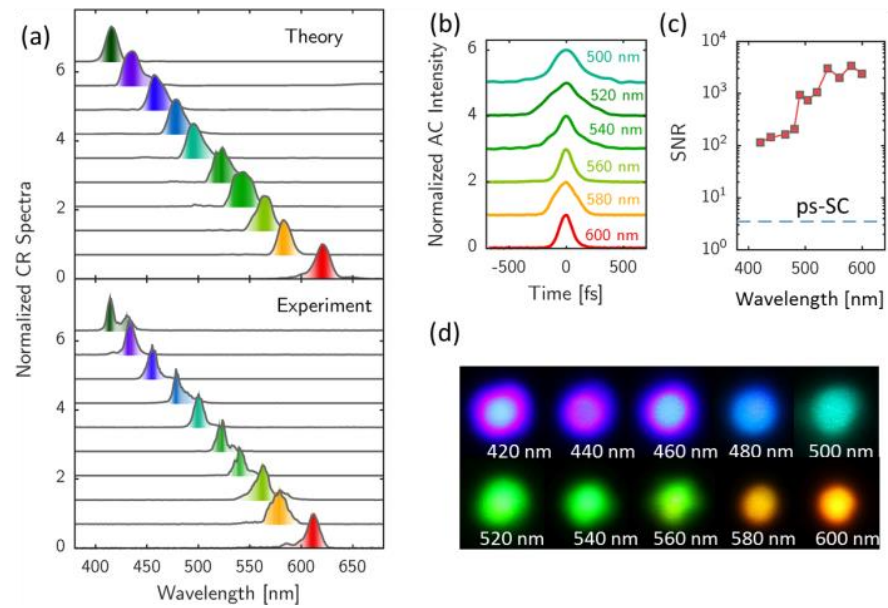

(d)

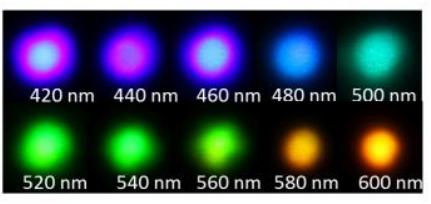

Fig. 2. Characterization of the tunable FOCR fiber laser. (a) Simulated and experimentally measured spectra of the generated tunable FOCR pulses. (b) Measured autocorrelation curves of the FOCR output. (c) The signal to noise ratio (SNR) of generated FOCR (red) and standard ps-SC source spectrally sliced with $10 \mathrm{~nm}$ bandwidth (FWHM) optical bandpass filters (blue). (d) The far-filed images of the generated FOCR pulses.

Laser intensity noise is an important parameter for the laser's bio-imaging applications. The signal-to-noise ratio (SNR) of the FOCR output is shown in Fig. 2(c). The results confirm that the tunable FOCR laser has 1-2 orders of magnitude better noise behaviour as compared to a standard 10-nm filtered ps-pumped supercontinuum sources (ps-SC). Therefore, for spectroscopy and bio-imaging applications, the time needed to acquire good data with the laser shown here can be 2 to 4 orders of magnitude faster as compared to the best current alternative. The measured far-field images of the pulses as shown in Fig. 2(d), indicating the single mode output of the generated FOCR signals, even though the PCF used is multimode at visible wavelengths.

In conclusion, we have developed a novel widely-tunable fiber laser technology based on FOCR in fiber tapers. Using this principle, continuous visible wavelength tunability over $200 \mathrm{~nm}$ of FOCR from a single tapered PCF is demonstrated. The short, $\sim 100 \mathrm{fs}$ pulse duration and excellent noise properties make such a laser system attractive for a great variety of applications, particularly in life sciences.

\section{References}

[1] N. Savage, Nat. Photonics 4, 124 (2010).

[2] J. M. Dudley, G. Genty, and S. Coen, Rev. Mod. Phys. 78, 1135 (2006).

[3] K. L. Corwin, N. R. Newbury, J. M. Dudley, S. Coen, S. a Diddams, K. Weber, and R. S. Windeler, Phys. Rev. Lett. 90, 113904 (2003).

[4] X. Liu, J. Laegsgaard, R. Iegorov, A. S. Svane, F. Ö. O. Ilday, H. Tu, S. A. Boppart, and D. Turchinovich, Photonics Res. 5, 750 (2017).

[5] G. Chang, L. L.-J. Chen, and F. X. Kärtner, Opt. Lett. 35, 2361 (2010).

[6] X. Liu, A. S. Svane, J. Lægsgaard, H. Tu, S. A. Boppart, and D. Turchinovich, Accept. by J. Phys. D Appl. Phys. (2015).

[7] W. Zheng, D. Li, Y. Zeng, Y. Luo, and J. Y. Qu, Biomed. Opt. Express 2, 71 (2011). 